livraisons

d'Histoire

de l'Architecture

\section{Livraisons de l'histoire de l'architecture}

14 | 2007

Piscines

\title{
La piscine de Pantin (1935-1937), une réalisation architecturale et sociale d'envergure
}

The swimming pool of Pantin: an ambitious architectural and social achievement

Der Bau des Schwimmbads von Pantin, ein architektonisch und sozial weittragendes Programm

\section{Christelle Inizan}

\section{OpenEdition}

Journals

Édition électronique

URL : http://journals.openedition.org/lha/424

DOI : $10.4000 /$ lha. 424

ISSN : 1960-5994

Éditeur

Association Livraisons d'histoire de l'architecture - LHA

Édition imprimée

Date de publication : 10 décembre 2007

Pagination : $39-53$

ISSN : 1627-4970

Référence électronique

Christelle Inizan, «La piscine de Pantin (1935-1937), une réalisation architecturale et sociale d'envergure », Livraisons de l'histoire de l'architecture [En ligne], 14 | 2007, mis en ligne le 10 décembre 2009, consulté le 01 mai 2019. URL : http://journals.openedition.org//ha/424 ; DOI : 10.4000//ha.424

Ce document a été généré automatiquement le 1 mai 2019.

Tous droits réservés à l'Association LHA 


\section{La piscine de Pantin (1935-1937), une réalisation architecturale et sociale d'envergure}

The swimming pool of Pantin: an ambitious architectural and social

achievement

Der Bau des Schwimmbads von Pantin, ein architektonisch und sozial

weittragendes Programm

Christelle Inizan

\section{Le socialisme municipal pantinois}

Les historiens se souviennent que, lors des élections municipales de 1919, vingt-quatre communes de la Seine banlieue furent remportées par la SFIO ${ }^{1}$. Tout l'arc de cercle des communes ouvrières jouxtant Paris passèrent ainsi du communisme au socialisme orthodoxe de la section française de l'internationale ouvrière ou à un socialisme plus indépendant tel que l'incarna de 1919 à 1938 le sénateur-maire Charles Auray à Pantin.

1 Curieusement, les équipements urbains voulus par Charles Auray sont longtemps restés dans l'ombre des réalisations architecturales entreprises par Henri Sellier, maire emblématique de Suresnes de 1919 à 1941, fondateur et président de l'office public du département de la Seine. Les deux hommes illustrent pourtant une même idéologie "municipaliste ", empruntée à la sociale-démocratie mise en place dans les capitales autrichienne et néerlandaise ${ }^{2}$. La longévité du mandat de ces deux maires et le chevauchement de leurs actions dans le temps constituent un autre de leurs traits communs. Ce mode inédit de gestion municipale se caractérise par la volonté politique nouvelle de déconcentrer l'action communale en sortant enfin de l'hôtel de ville, lieu de la représentation municipale et de ses prestations publiques. Par la création de nouveaux services collectifs communaux et le redéploiement d'équipements de proximité au pied des cités, le « socialisme municipal » trouve là son type d'expression privilégiée. 
Charles Auray, qui remplaça le maire radical Charles David dont l'équipe était majoritairement constituée d'industriels et de notables, s'employa durablement à modifier la morphologie de cette ville ouvrière en engageant de gros travaux urbains, proposant du même coup à ses administrés quantité d'emplois dans le bâtiment. En ces années de rigueur économique et de pertes d'emplois, sur fond de conflits politiques aigus, les grandes entreprises sociales menées par les élus offraient de salvateurs appels d'air pour la population locale. La municipalité de Pantin pronostiquait que la réalisation d'une piscine sur la commune permettrait à elle seule l'emploi, sur un chantier d'environ sept mois, d'un effectif de 100 à 150 chômeurs, et de 25 à 30 personnes pour l'exploitation proprement dite de la piscine ${ }^{3}$

Aussi Pantin vit-elle fleurir en quelques années, au sud d'abord, sur les coteaux, les premières habitations à bon marché, une innovante école de plein air destinée à accueillir les enfants souffrant de maladies respiratoires, un stade et une maison de retraite. Ces réalisations furent complétées par une cité jardin rue des Pommiers ${ }^{4}$. Parallèlement, un certain nombre de quartiers furent dotés d'équipements spécifiques tels que bainsdouches ou crèches. La création d'une piscine près de la mairie vint parachever ces programmes d'équipements municipaux.

\section{Les années trente : une vague constructive de piscines}

Offrir des installations sportives dans une vision hygiéniste idéale, tel est le credo de nombreux nouveaux maires de banlieue pour qui le sport est une pièce importante du dispositif social et politique. En 1924, les jeux olympiques d'été de Paris avaient mis en lumière le retard pris en France, tant en matière de pratiques sportives que d'équipements, au regard des pays du nord notamment l'Angleterre et l'Allemagne. La "VIII ${ }^{e}$ Olympiade de l'ère moderne » avait néanmoins permis l'édification à Paris, dans le $\mathrm{XX}^{\mathrm{e}}$ arrondissement, de la piscine des Tourelles - premier bassin découvert de 50 mètres - où brilla le médaillé d'or Johnny Weissmuller.

2 Des revues spécialisées se firent bientôt le relais de ce mouvement en faveur de l'architecture du sport. L'Architecture d'aujourd'hui (AA), dont le premier numéro paru en novembre 1930, est à la pointe de cet engagement. Le numéro 3 d'avril 1934 est ainsi entièrement consacré aux constructions sportives et une large place occupée par les piscines et plages. La même année, Charles-Edmond Sée publie dans La Construction moderne une série de réflexions sur les piscines. La revue propose en conclusion le plantype d'une piscine élaboré à la demande de la fédération française de natation, par les architectes Chollet et Mathon ${ }^{5}$. «Dans les années 1920-1930, la plupart des maîtres d'œuvre d'édifices sportifs pratiquèrent une architecture intégrée à la ville, au quartier, au lotissement ${ }^{6}$.» Les architectes urbanistes et hygiénistes qui partagent ce goût de l'équipement public et de l'œuvre sociale sont alors moins soucieux d'élaborer un style que de penser la construction sportive d'une manière neuve ${ }^{7}$. Certains ensembles jouent la polyvalence, d'autres sont intégrés à des bâtiments administratifs ${ }^{8}$. Une troisième voie consiste à les associer à d'autres constructions. Le plus adaptable de ces équipements sportifs est sans conteste le gymnase. Ce dernier peut en effet se loger facilement dans presque tous les types de bâtiments publics.

En matière de piscine, les combinaisons architecturales sont moins aisées. Le plus souvent, ce sont les bains-douches qui y sont intégrés ; ainsi, la piscine de la Butte aux 
Cailles à Paris XIII (1922-24) de Louis Bonnier, celle de Jeumont (1926) ou de Carpentras (1931), celle de Bègles (1930-32). On la trouve également associée à des casinos : casino d'Hossegor (1927-28), casino Balneum de Dinard (1928), aujourd'hui démoli, et plus largement à des stades comme la piscine de plein air de Bruay-la-Buissière (1931), la piscine judaïque de Bordeaux (1934-35) de Léon Madeline. Pour sa part, le groupe scolaire Paul Langevin à Suresnes (1927) de Payret-Dortail accueille en son centre un gymnasepiscine. Dans la même ville, une configuration identique se retrouve à la cité-jardin, conçue par les architectes Maistrasse, Quoniam, Dumail, Bazin. Quant au groupe scolaire Marius Jacottot de Puteaux (1933-1938) des frères Niermans, il présente l'exceptionnel avantage d'intégrer une piscine à l'ensemble.

La plus insolite des compositions reste cependant la piscine-usine de traitement d'ordures ménagères de la Butte rouge à Châtenay-Malabry, dans les Hauts-de-Seine. La combustion des déchets de la cité-jardin servait à chauffer l'eau de la piscine.

Assainissement et pratique sportive sont ici réunies avec audace dans un bâtiment double, conçu et réalisé entre 1931 et 1935 par les architectes Bassompierre, de Rutté et Sirvin.

\section{Pantin, un projet mixte : sportif et industriel}

La réalisation de Châtenay-Malabry - première piscine couverte construite dans la banlieue parisienne - à la pointe de l'innovation et de l'économie, ne peut avoir été ignorée par l'équipe municipale de Pantin lorsqu'elle conçut, en 1936, son propre projet de piscine, projet qui s'est imposé par la décision de la compagnie générale des eaux (C.G.E.) de créer dans la ville un point d'eau alimenté par des puits. En édifiant une piscine à proximité, on faisait ainsi coup double en l'alimentant directement avec les eaux les plus profondes de ces forages, eaux à la fois chaudes et moins calcaires. Le terrain que la C.G.E. immobilisa pour la création de son usine et de ses installations de pompage faisait partie d'une propriété de deux hectares environ sur lesquels $6000 \mathrm{~m}^{2}$ restaient disponibles ${ }^{9}$. Cette parcelle, occupée par des ateliers et des magasins divers, appartenait à la société Félix Potin, fut bientôt acquise par la commune ${ }^{10}$. Sa situation, proche à la fois de l'hôtel de ville et de la gare, en bordure des voies de chemins de fer du réseau Est, se prêtait particulièrement bien à la construction d'une piscine municipale et à l'aménagement d'une plaine de jeux dont les accès se trouvaient dans le prolongement des cours de deux écoles limitrophes ${ }^{11}$.

Le maire de Pantin décida de confier à son fils Charles, pour un budget de quatre millions de francs de l'époque - acquisition du terrain comprise -, la construction de la piscine. Au bassin de natation, pouvant recevoir des compétitions sportives, vinrent s'ajouter d'autres équipements : un terrain de sports et des salles de culture physique implantées au dernier étage de la piscine. La ville ne pouvant se procurer les fonds nécessaires à l'exécution du projet sollicita un emprunt sur trente ans auprès de la caisse de crédit aux départements et aux communes ${ }^{12}$. La commande s'élargit à l'usine des eaux ainsi qu'au pavillon d'habitation destiné au directeur de l'usine, situé entre les deux édifices.

Charles Auray, alors jeune architecte de vingt-quatre ans, trouve là l'occasion de signer sa première œuvre, tandis que son père justifiait son choix par l'économie des honoraires $\mathrm{du}$ maître d'œuvre ainsi dégagée. À l'architecte débutant est adjoint un ingénieur expérimenté de la C.G.E., Jean Molinié. Le tandem ainsi constitué prit en charge 
l'ensemble de l'opération. Les travaux commencèrent le 15 juin 1936 et s'achevèrent un an plus tard.

Architecte diplômé par l'État (DPE) en 1937, Charles Auray fut l'élève de Jean Trouvelot et de Georges-Henri Pingusson qui écrivit en 1934 un article remarqué donnant des «Renseignements utiles pour l'étude d'une piscine $»^{13}$. Auray a travaillé chez Florent Nanquette ${ }^{14}$, architecte spécialisé dans les programmes sociaux et actif à la périphérie de Paris notamment à Montreuil et à Pantin où il est le maître de l'œuvre de l'école de plein air (1932-1933) et de la maison de retraite (1935). Charles Auray a probablement participé à l'élaboration de ce dernier projet avant de se voir confier la construction de la piscine municipale et de l'usine des eaux. Après la guerre, Charles Auray n'eut malheureusement plus l'occasion de réaliser de projets aussi librement. Il construisit des logements sociaux et des lycées, esclave désormais « de la trame 180 et des normes réduites ». Charles Auray interrogé en 1996 lors de la procédure de protection au titre des monuments historiques s'exprima en ces termes : «La piscine et l'usine des eaux de Pantin conservent une place privilégiée dans ma carrière ${ }^{15}$."

7 Molinié et lui apportèrent beaucoup de soin à la réalisation de ce double programme, fortement inspirée de l'architecture néerlandaise. Du reste, peu avant sa commande, Charles Auray a effectué un voyage aux Pays-Bas pour étudier l'architecture épurée de Willem Marinus Dudok, influencé par Frank Lloyd Wright et le mouvement De Stijl. L'hôtel de ville d'Hilversum, construit entre 1928 et 1932, deux écoles d'Hilversum et le magasin Bijenkorf de Rotterdam (ill. 1) servirent en partie de source d'inspiration aux deux constructions de Pantin ${ }^{16}$.

III. 1 : Façade nord-ouest de l'hôtel de ville d'Hilversum en 1931

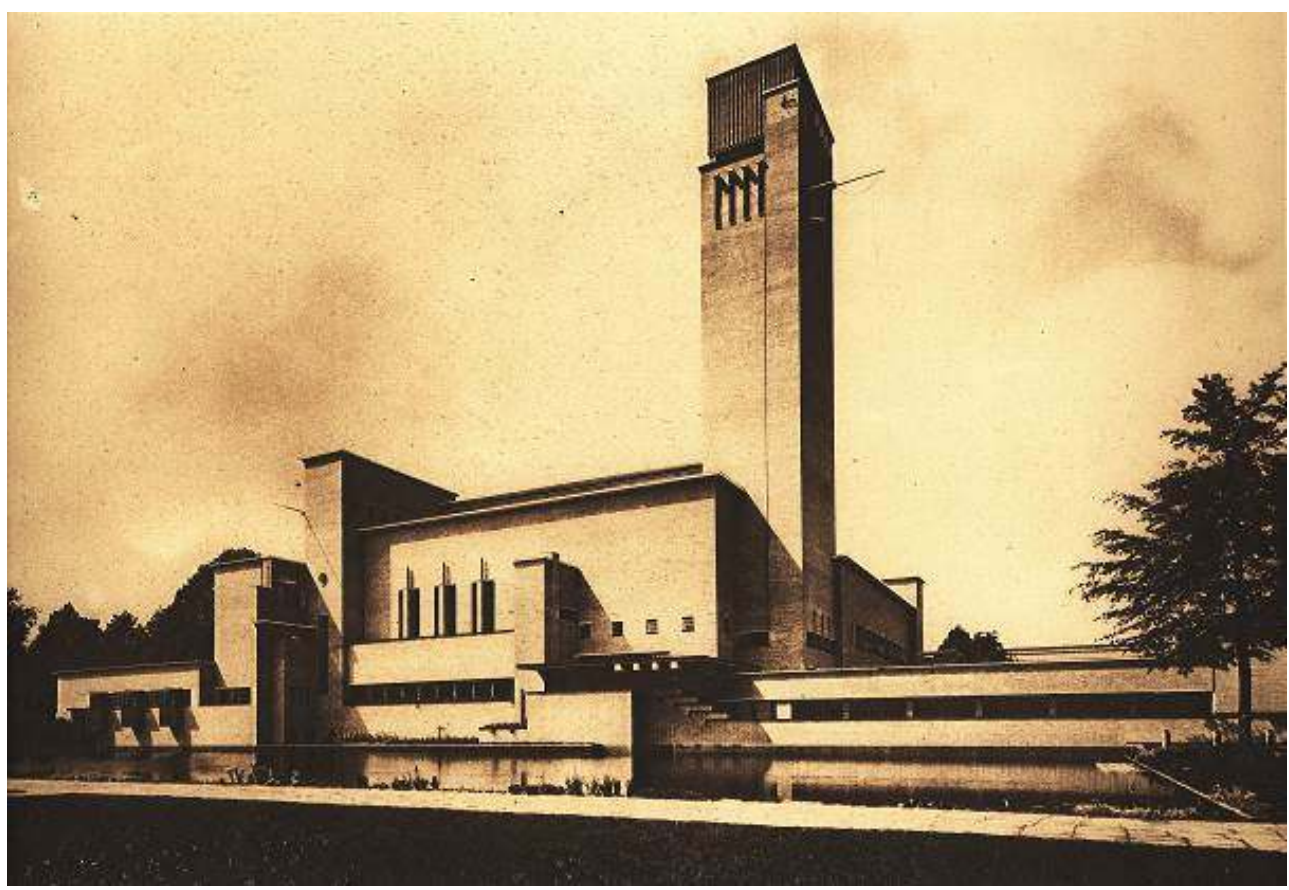

L'Architecture d'aujourd'hui, mars 1932

Cliché de l'auteur

D'autres architectes français (Mathon et Chollet à l'hôtel de ville de Cachan en 1938, Dubreuil et Hummel pour le groupe scolaire de Maisons-Alfort entre 1930 et 1934, 
Lhôtelier et Robin au poste de coupure de la société Nord-Lumière à Aubervilliers en 1939) firent également référence à ces édifices insignes, internationalement reconnus, et publiés dans L'Architecture d'aujourd'hui de mars $1932^{17}$. La leçon de Dudok sera retenue : fort ancrage du bâti dans son environnement, jeu exclusif des masses, volumes horizontaux, fenêtres basses en ruban, toit plat, soin extrême apporté aux détails et décoration reléguée aux espaces intérieurs.Pour sa part, Françoise Hamon voit une parenté très nette entre «la piscine de Pantin et le gymnase-tribune d'André Lurçat à l'école Karl Marx de Villejuif : même esthétique radicale du bunker, efficace et impressionnante, absence de tout ornement, rationalité nue ${ }^{18}$.

\section{L'architecture de la piscine}

9 La piscine constitue évidemment le bâtiment phare de cet ensemble urbain. Elle domine par sa masse imposante la totalité de la composition (ill. 2).

\section{2 :Le magasin Bijenkorf de Rotterdam}

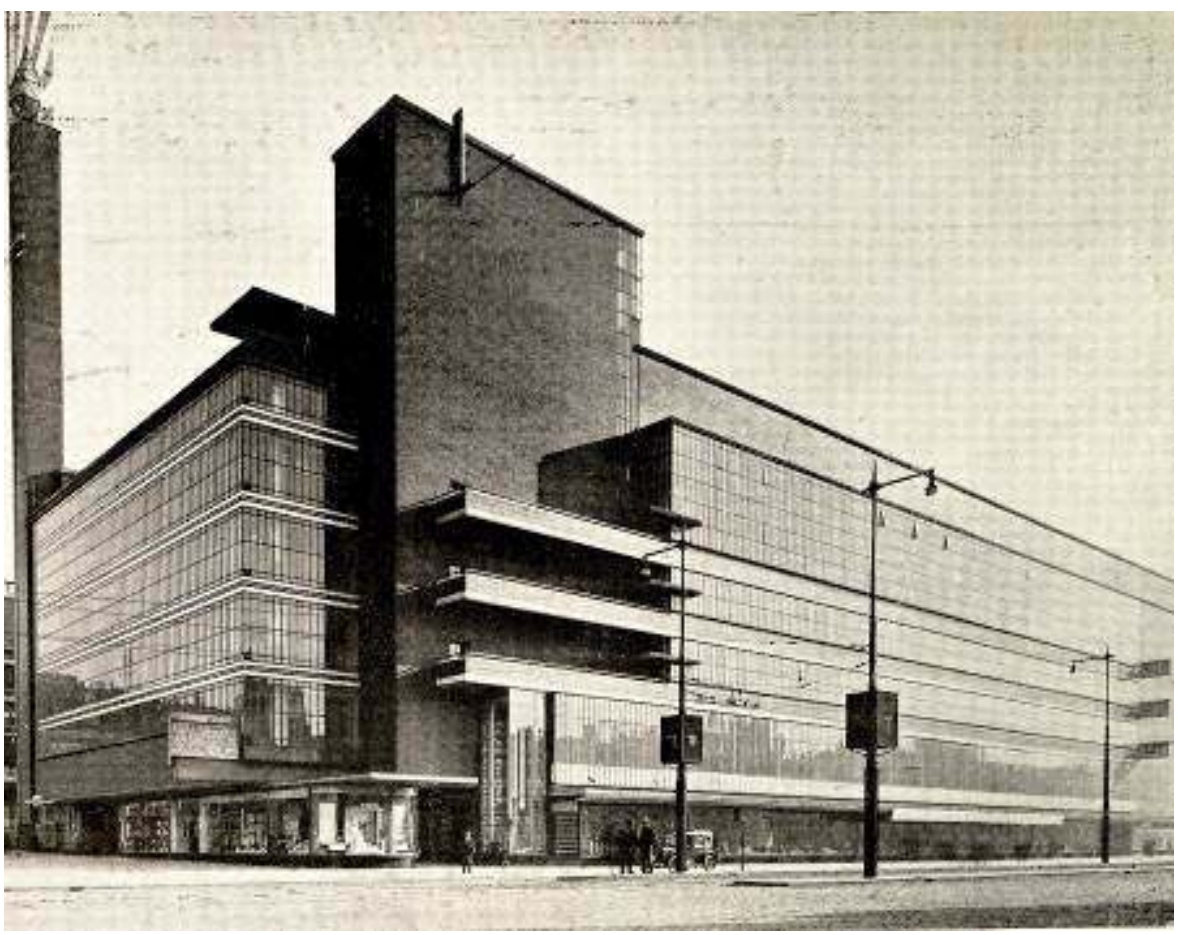

L'Architecture d'aujourd'hui, mars 1932

Cl. de l'auteur

D'un côté, se trouve la parcelle du point d'eau, autrefois jalonnée de plusieurs pavillons bas, de l'autre, un terrain plat de $4000 \mathrm{~m}^{2}$ qui sépare la piscine du groupe scolaire Sadi Carnot construit en 1889. Cette surface a été aménagée en terrain de jeux avec abris, procurant ainsi à l'école l'espace qui lui manquait. Un garage à bicyclettes à ossature et toiture en béton armé, hourdis de briques, construit à proximité de la piscine, complète l'agencement général. Les murs de clôtures et les portails sont également élevés en briques de parement.

Le toit plat de la piscine et sa façade d'entrée en retrait d'alignement de l'avenue confèrent au bâtiment monumentalité et représentativité. Cette disposition semble avoir 
été inspirée par le stade municipal de Courbevoie réalisé en 1930 par Nanquette ; comme en témoigne le premier projet d'élévation des façades de la piscine de Pantin daté d'octobre 1935 et conservé aux archives communales. Néanmoins, la silhouette de blockhaus de la piscine de Pantin renvoie une nouvelle fois aux réalisations de Dudok. Il n'est pas inintéressant de savoir que l'architecte avait travaillé, entre 1910 et 1913, à un projet de bunker ${ }^{19}$. La filiation néerlandaise est renforcée par l'utilisation en revêtement de la brique rouge des Comptoirs tuiliers du Nord, matériau par excellence de la banlieue, économique et facile à transporter.

Le remplissage entre les éléments de l'ossature en béton armé est exécuté, à l'extérieur, en briques de parement apparentes et en briques de remplissage vers l'intérieur ${ }^{20}$. Du même fabricant proviennent les éléments en grès émaillé noir qui couronnent l'édifice et encadrent les baies. Les joints horizontaux sont nettement accusés, largement creusés, tandis que l'appareillage a fait disparaître les joints verticaux (ill. 3).

\section{3 :Le magasin Bijenkorf de Rotterdam}

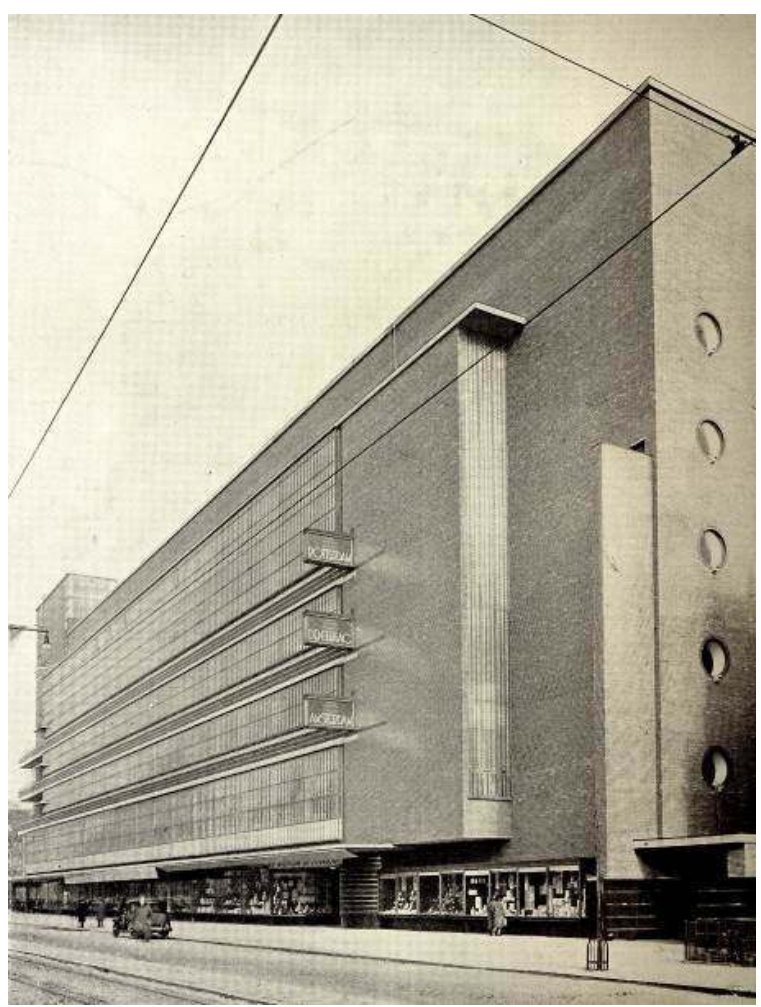

L'Architecture d'aujourd'hui, mars 1932

Cl. de l'auteur

L'architecte a voulu rompre l'imposante masse de l'édifice par des effets de volumes à différents niveaux, en jouant sur des avancées et des retraits de travées qui viennent briser le rythme horizontal des baies ${ }^{21}$. La verticalité rigoureuse de la cheminée surmontée d'un aérateur en béton armé et placée à l'arrière du bâtiment, contribue à cet effort (ill. 4). 


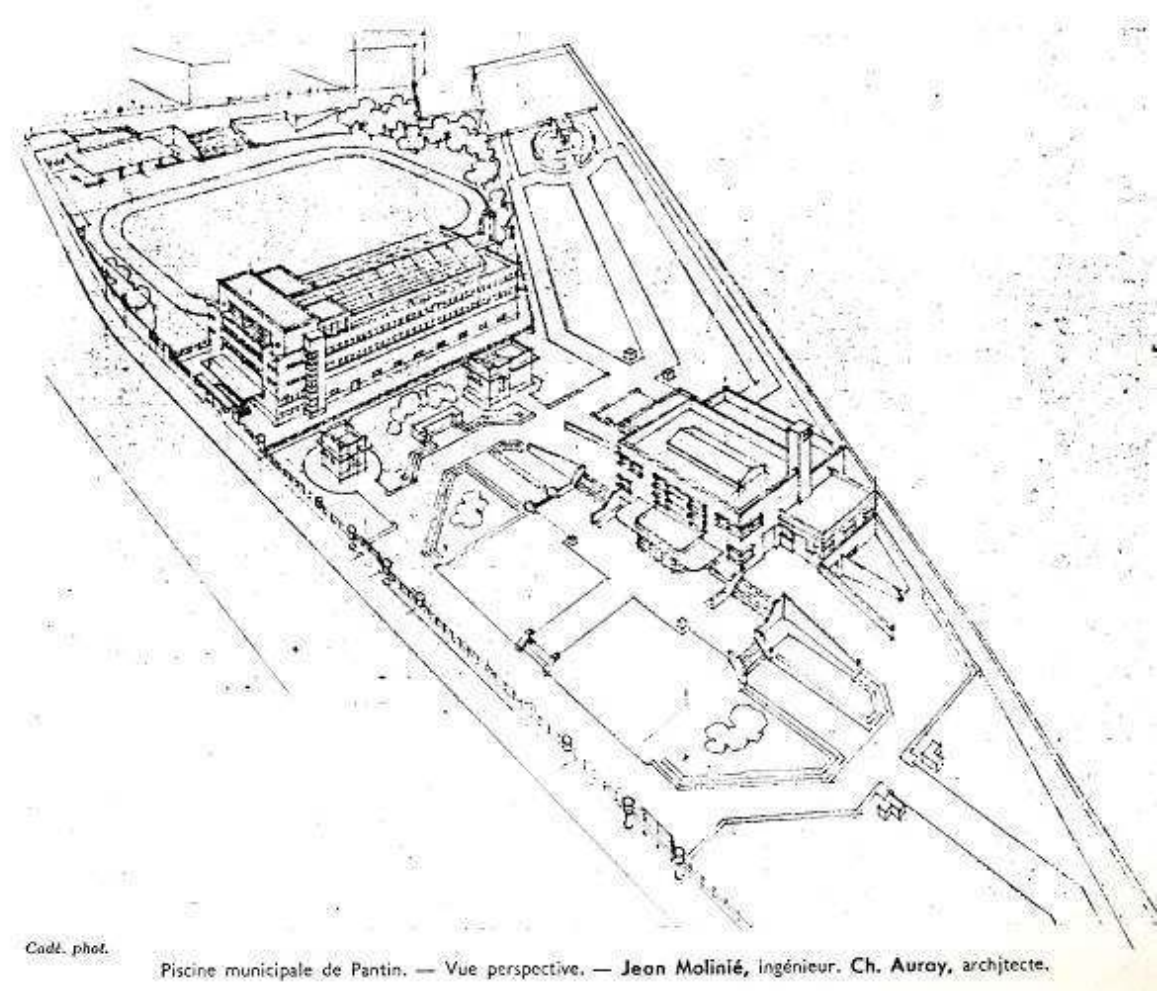

La Construction moderne, 6-13 nov. 1938

Cl. de l'auteur

Les châssis métalliques pivotants des fenêtres rondes rappellent les hublots d'un navire, mais également la fonction aquatique de l'édifice. La différence de taille des caractères formant les inscriptions de la façade, participe à cette volonté d'allègement du bâtiment. Davantage encore que la façade principale, les façades latérales et antérieure de la piscine évoquent celles du grand magasin de Rotterdam et, de façon moindre, la façade sud-est de l'hôtel de ville d'Hilversum dont la rigueur atténuée se retrouve dans le traitement différencié de l'aile de l'usine de Châtenay Malabry ou la façade arrière de la piscine parisienne de la rue Édouard-Pailleron (édifiée en 1934).

L'entrée de la piscine se fait par un emmarchement conduisant à un porche abrité22. Un large perron, divisé en son milieu par un volume convexe, sorte de loggia en fer à cheval dissimulant intérieurement des bancs fixes, sépare l'entrée de la sortie. Autour du hall, très lumineux grâce à ses baies supérieures horizontales, sont répartis plusieurs locaux : le logement du gérant (avec loge, salle à manger et cuisine au rez-de-chaussée, chambre au sous-sol), le cabinet médical et l'infirmerie, le bureau du directeur ${ }^{23}$. La caisse de forme arrondie, située dans l'axe de la loggia, surveille à la fois les allées et venues côté vestibule et, par une vitre, le bassin. De part et d'autre de la caisse, deux entrées débouchent sur un espace délimité par des barrières, faisant face au bassin de natation. Deux escaliers latéraux partiellement hors œuvre, dont les cages sont éclairées par de hautes verrières d'angle, conduisent les visiteurs, encore chaussés, aux étages où se trouvent des déshabilloirs collectifs pour les écoles et les groupes, de simples cabines réparties sur tout le pourtour du bassin, et de plus spacieuses placées à l'avant du bâtiment. Les arrivants empruntent ensuite "pour descendre à la plage » un escalier double situé à l'arrière du bâtiment, également vitré. Ce parcours hygiénique imposé les 
oblige à passer au rez-de-chaussée par les douches de propreté (il y a quinze douches côté hommes et quinze douches côté dames) en communication directe avec le trottoir des baigneurs (ill. 5).

\section{5 : Façade principale de la piscine de Pantin}

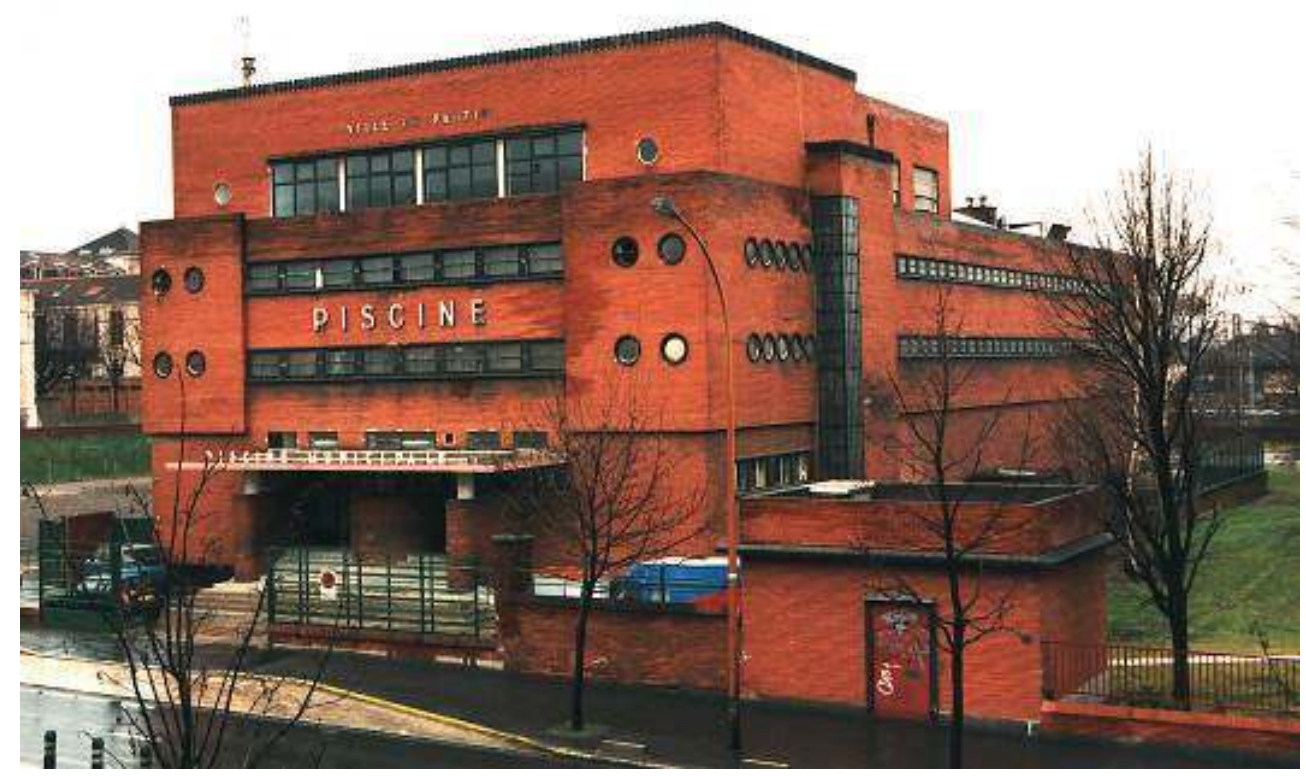

Cl. de l'auteur, CRMH Île-de-France, 1996

12 Le type adopté pour l'intérieur de la piscine est celui qu'avait mis en avant quelques années auparavant, à Paris, l'ingénieur Lucien Pollet, architecte de la célèbre piscine de la rue de Pontoise, de la rue de la Jonquière et de la rue Édouard-Pailleron.

Le bassin - petit et grand bains - de 33 mètres, aux normes de compétition, est éclairé par une verrière zénithale de même surface et entouré des quatre déshabilloirs communs et des deux cents trente-deux cabines de déshabillage individuel réparties sur deux niveaux auxquelles on accède par des galeries surplombant le bassin ${ }^{24}$. Comme les escaliers, ces cabines prennent jour en façades. Le dispositif théâtral est complété par un mur, initialement parabolique, véritable fond de scène, qui supporte l'horloge et accueillait autrefois le plongeoir à étages avec tremplin. Cet écran recouvert d'un granito bleu accroche le regard et axe toute la composition. Il contribue à souligner les silhouettes des plongeurs ainsi que les poutres de rive et les garde-corps des galeries-balcons (ill.6). 


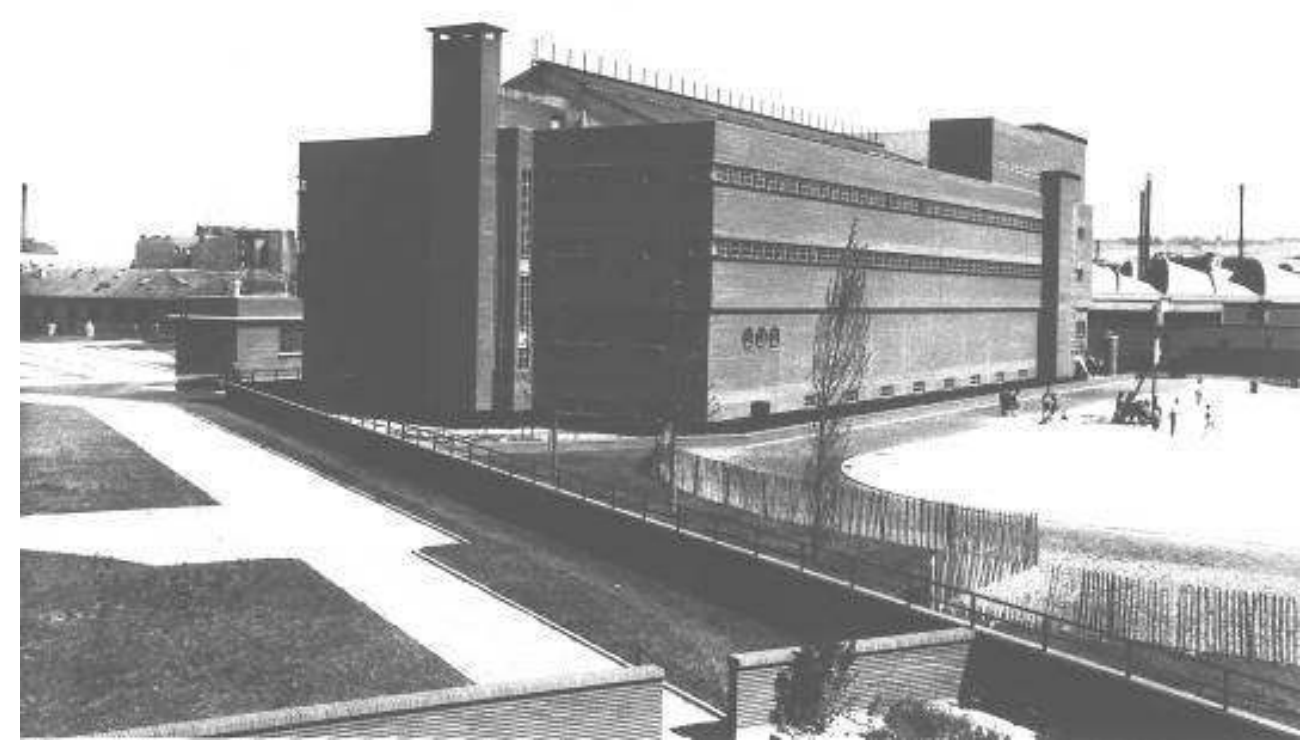

$\mathrm{Cl}$. Archives municipales de Pantin.

Un soin extrême a été apporté à tous les détails de l'aménagement intérieur. La tonalité générale des revêtements intérieurs est le blanc. À l'origine, la plupart des sols étaient carrelés " en mosaïque de hasard » - céramique en opus incertum - en grès cérame beige clair et disposés pour recueillir par des caniveaux sous caillebottis les eaux de ruissellements. Les parois verticales autour du bassin étaient tapissées jusqu'à hauteur de soubassement de carreaux de faïence blanche, notamment pour les banquettes des plages ${ }^{25}$, tandis que le reste du revêtement (les piliers de soutènement à mi-hauteur et le sol des plages) était constitué d'éclats de faïence blanche avec semis de mosaïque bleue bordée par des carreaux rehaussés d'émaux de Briare bleu. La cuve du bassin était également tapissée de faïence cassée à l'exception du brise-vague où les carreaux étaient entiers afin de faciliter le nettoyage par aspiration. Cinq lignes de nage en faïence bleu foncé ornent toujours le fonds du bassin, refait depuis.

16 Comme toujours, l'indépendance est complète entre la cuve du bassin et l'ossature de l'édifice. La cuve est sur poteaux. Elle a été construite en béton Portland, tassé par vibration. L'intérieur est enduit par projection au mortier. En cas de compétition sportive, le plan d'eau pouvait être remonté de 0m25 au moyen d'un système spécial. Audessus de l'entrée, de larges encorbellements accueillaient les spectateurs souhaitant assister aux compétitions. L'accès aux galeries-balcons était condamné par un système de portillons isolant les visiteurs des nageurs.

17 La toiture vitrée est à double pente constituée de fermes métalliques légères permettant une ventilation effective et un bon éclairage zénithal. Le plafond proprement dit est en dalles de verre démontables. Elles sont posées librement sur un système de croisillons de fers en T. Un pont roulant permet d'opérer le nettoyage des verres et de placer des appareils d'éclairage du bassin lors des épreuves sportives. En temps normal, il est placé 
contre le pignon opposé à l'entrée principale. Une porte pratiquée dans ce pignon permet de passer de la cabine à la terrasse. bâtiment, est éclairée par les bandeaux horizontaux des baies et les deux oculi de la façade principale. De petites pièces secondaires se greffent à cette grande salle : vestiaires avec douches, salle de gymnastique médicale, salle d'escrime et de boxe, cabinets de mensuration ${ }^{26}$.

Au sous-sol, les installations sont groupées : la chaufferie près du système de ventilation, l'équipement électrique avec le réseau sanitaire (traitement de l'eau et appareils de régénération de l'eau ${ }^{27}$ ).

Ouverte au public en mai 1937, la piscine de Pantin connut un succès considérable : 230 000 entrées. La municipalité contribua largement à cette réussite en finançant une vaste campagne publicitaire de lancement dans les journaux et par affichage tout en promouvant Jean Taris, champion d'Europe de natation, directeur de l'établissement. Le tarif très bas du prix d'entrée a également contribué à ce succès ${ }^{28}$. La piscine fut utilisée dès son ouverture par les élèves du collège qui bénéficiaient d'un accès gratuit (ill. 7).

\section{7 : Plans de l'étage et du rez-de-chaussée}

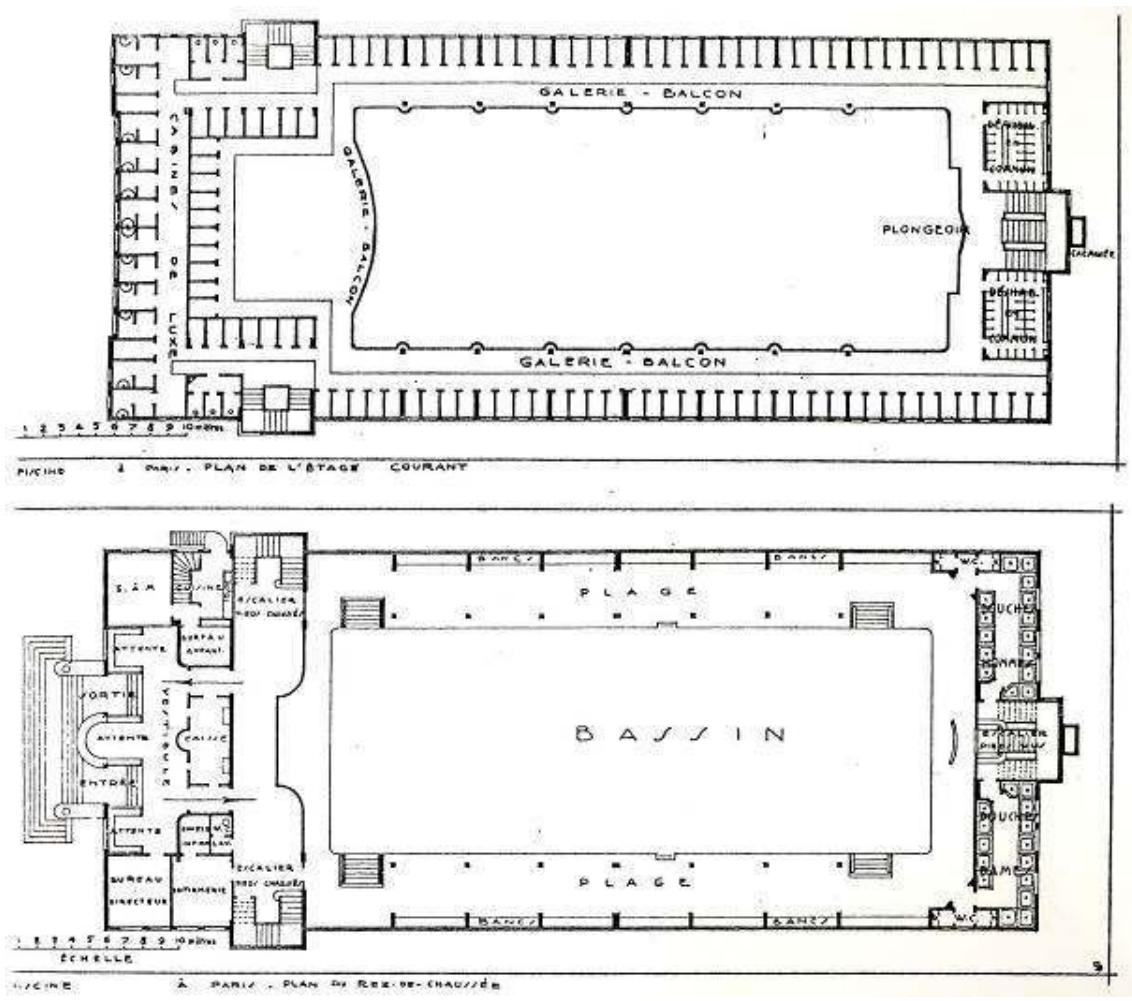

La Construction moderne, 6-13 nov. 1938

Cl. de l'auteur

21 Ceux-ci durent cependant attendre octobre 1948 avant d'utiliser la salle de culture physique $^{29}$, disponible seulement pendant les jours de pluie de la période d'hiver, la culture physique se pratiquant habituellement en extérieur, sur le terrain de sport. Malgré ce succès populaire les critiques ne manquèrent pas. Les communistes dénoncèrent vivement la gestion du maire de Pantin et « les tromperies de son action en 
faveur du sport », le premier magistrat encourageant selon eux « le sport spectacle » en dépit de son engagement à gauche ${ }^{30}$.

\section{L'usine élévatoire et de traitement des eaux}

La station de pompage et de production d'eau souterraine de Pantin est implantée à proximité immédiate de la piscine mais très en retrait, à l'arrière des bassins de stockage. Un premier puits artésien profond d'environ 800 mètres avait d'abord été foré. L'eau obtenue, bactériologiquement pure, peu chargée en sels minéraux, atteignait environ $30^{\circ} \mathrm{C}$. Une eau de cette température convenait parfaitement à l'alimentation d'une piscine mais ne pouvait être distribuée sans correction aux abonnés. C'est la raison pour laquelle trois autres puits, moins profonds, furent forés dans une autre nappe aquifère, à 100 mètres de profondeur, procurant une eau froide.

Á l'origine, de petits pavillons disséminés sur le terrain du point d'eau abritaient ces différents forages. Ces constructions dispersées étaient apparentées par leur style. Seul le pavillon le plus important subsiste. Il abrite l'usine de pompage et de déferrisation. Construit entre 1935 et 1936, en même temps que la piscine, il en épouse l'architecture (baie centrale, fenêtres d'angle, bandeau). La petite usine domine un monticule gazonné qui cache un réservoir semi-enterré où se mélangent les eaux. Une grille sépare toujours le pavillon et le grand espace traité en jardin de la voie publique.

Sous un châssis vitré, dans un ronronnement continu, quatre moteurs pompaient l'eau dans les nappes phréatiques. Certaines machines élévatoires d'époque subsistent. Des cinq puits de forage d'origine, un seul fonctionne aujourd'hui. « Cette eau de très bonne qualité est déferrisée puis chlorée avant d'être stockée dans deux réservoirs enterrés. Les $150 \mathrm{~m}^{3}$ d'eau potable traités par heure sont utilisées pour alimenter une partie de la région de Pantin. L'usine fonctionne automatiquement et à distance à partir du poste de commande de Neuilly-sur-Marne ${ }^{31}$. »

L'usine de forage, aujourd'hui propriété du syndicat des eaux d'Île-de-France et exploitée par la C.G.E., a été soumise depuis sa création à bon nombre d'aléas de fonctionnement et a subi certaines transformations architecturales, au demeurant habilement menées: suppression de l'avancée centrale et du vestibule d'entrée, installation de claustras en brique dans les baies, encadrements des fenêtres peints en noir dans l'esprit chromatique de la piscine (ill. 8). 
III. 8 : Volume intérieur de la Piscine

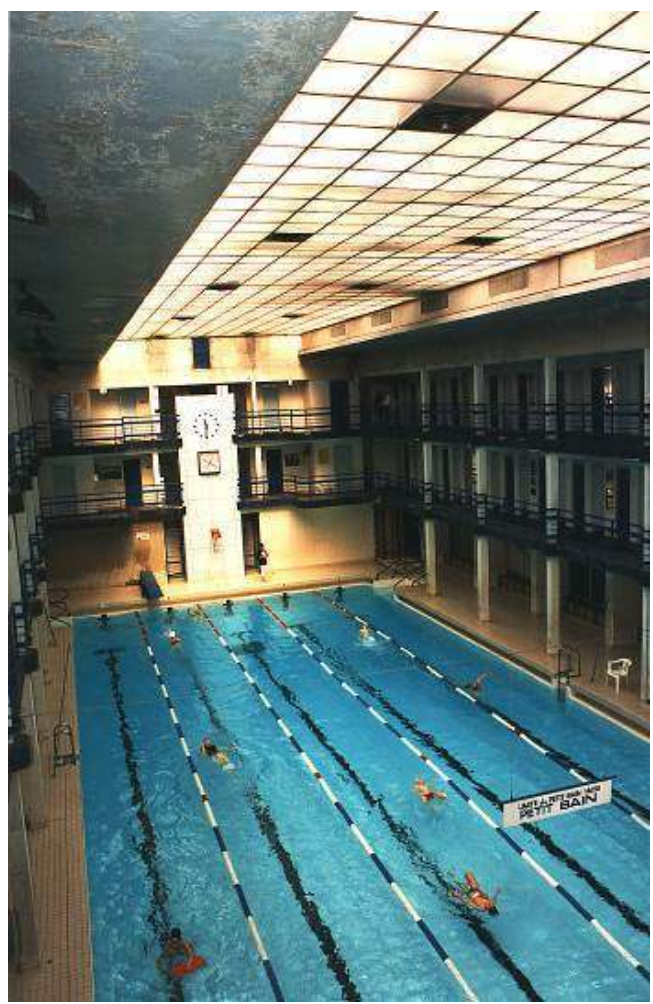

Cl. de l'auteur, CRMH Île-de-France, 1996 qu'elle ne l'était à l'époque ${ }^{32}$. À cet ensemble sportif et industriel, était associé un petit pavillon d'habitation (la maison du directeur de l'usine), de facture très soignée, qui récapitulait en tout point les grandes options stylistiques des deux édifices manifestes ${ }^{33}$. Cette construction a été malheureusement démolie en 1994.

En 1937, Charles Auray se vit de nouveau confier la construction d'une petite usine de la C.G.E. à Épinay-sur-Seine fortement inspirée de sa première réalisation ${ }^{34}$. Dans le paysage de Pantin, la piscine et l'usine élévatoire des eaux constituent un élément fort du paysage. Ils s'insèrent magnifiquement dans le site de la mairie. La brique sert de trame à ce subtil jeu visuel où les deux édifices des années trente répondent à l'école voisine, plus ancienne. L'espace alentour met tout particulièrement en valeur cette architecture peu altérée par le temps, renvoyant au concept néerlandais primitif, soucieux d'une symbiose entre environnement et bâti. L'ensemble de Pantin apparaît comme un témoignage particulièrement intéressant des grands équipements urbains de la petite couronne, illustrant l'une des grandes périodes de l'architecture utilitaire et sociale en France, 1925-1935. La piscine de Pantin "l'une des plus modernes de la région parisienne " ${ }^{35}$ fait partie de ces bâtiments méconnus qui ont contribués à la notoriété d'une banlieue progressiste et audacieuse. 


\section{NOTES}

1. http://biosoc.univ.-paris1.fr/histoire/lieux/departmt/soc1.htm, loc. cit. Jean-Paul Brunet, Le Socialisme en banlieue nord et nord-est de Paris de 1945 à nos jours, table ronde de l'AHMO aux arch. dép. 93, le 13 décembre 2001, introduction..

2. http://www.Ville-pantin.fr/download/pdf/ logement, loc. cit. Daniel Tajan, Le Logement social, parcours d'architecture du 3 décembre 2000.

3. Arch. mun. Pantin, M21, Rapport de lutte contre le chômage. Construction d'un bassin municipal à Pantin. Exposé des principaux motifs qui ont poussé la municipalité à envisager la réalisation de ce projet, s.d., 2 p.

4. http://www.ville-pantin.fr/fileadmni/MEDIA/Histoire de Pantin, consulté en mai 2007, p. 5.

5. Antoine Le Bas, Architectures du sport - 1870-1940, Val-de-Marne, Hauts-de-Seine, Cahiers de l'Inventaire $n^{\circ} 23$, Paris, éd. Connivences, 1991, p. 62.

6. Antoine Le Bas, Architectures du sport [...], op. cit., p. 63.

7. La Construction moderne, $1^{\mathrm{er}}$ avril 1934, p. 463-464.

8. Antoine Le Bas, Architectures du sport [...], op. cit., p. 63.

9. Arch. mun. Pantin, M21, délibération du conseil municipal, séance du 30 octobre 1935.

10. Arch. mun. Pantin, M21, promesse de vente du $1^{\mathrm{er}}$ décembre 1935.

11. Arch. mun. Pantin, M21, plan aquarellé d'aménagement du point d'eau de la C.G.E. et de la piscine municipale, 30 oct. 1935.

12. Arch. mun. Pantin, M21, délibération du conseil municipal, séance du 30 octobre 1935.

13. L'Architecture d'aujourd'hui, $\mathrm{n}^{\circ} 3$, avril 1934, p. 56-57.

14. Paul Chemetov, Bruno Dumont, Bernard Marrey, Paris-banlieues 1919-1939, Paris, Dunod, 1989, p. 217.

15. Témoignage oral.

16. Ministère de la culture, direction régionale des affaires culturelles d'île-de-France, dossier de recensement de la conservation régionale des monuments historiques, Christelle Inizan, juin 1996.

17. L'Architecture d'aujourd'hui, mars 1932, $\mathrm{n}^{\circ} 2$.

18. Antoine Le Bas, Architectures du sport, op. cit., p. 15.

19. Emma Lavigne, Le Collège néerlandais, Dudok: la leçon de l'architecture militaire, mémoire de DEA, Paris I, sous la direction de Gérard Monnier, oct. 1996, p. 12.

20. Arch. mun. Pantin, M21, cahier des charges, conditions générales de l'appel à la concurrence, s.d., $34 \mathrm{p}$.

21. Arch. mun. Pantin, M21, élévation de la façade latérale, s.d.

22. Arch. mun. Pantin, M21, élévation de la façade principale, s.d.

23. Arch. mun. Pantin, M21, plan du r.d.c., oct. 1935.

24. Arch. mun. Pantin, M21, plan des étages, coupes longitudinale et transversale, oct. 1935, La Construction moderne, 6-13 nov. 1938, ill. p. 60, 62.

25. La Construction moderne, 6-13 nov. 1938, p. 62.

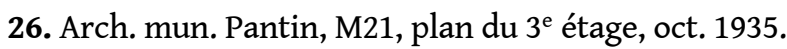

27. Arch. mun. Pantin, M21, plan du sous-sol. 
28. Arch. mun. Pantin, M21, lettre de Lafaille, exploitant de la piscine à un conseiller municipal, 1951.

29. Arch. mun. Pantin, M21, inauguration de la salle de culture physique, photographie ancienne, s.d.

30. http://www.wearefootball.org/PDF/le-football-ouvrier-en-region-parisienne.pdf, loc. cit. Nicolas Ksiss, Société et représentations, revue publiée par l'université de Paris XIII, 1998.

31. CAUE 93 et DRAC Ile-de-France, Le Patrimoine du XXe siècle, 100 visites pour 100 monuments, septembre 2000, 100 fiches.

32. Paul Chemetov, Bruno Dumont, Bernard Marrey, Paris-Banlieues, op. cit., p. 188-189.

33. Arch. mun. Pantin, M21, pavillon d'habitation du directeur de l'usine, photographie ancienne, v. 1945 ; La Construction moderne, 6-13 nov. 1938, ill. p. 60.

34. L'Architecture d'aujourd'hui, $\mathrm{n}^{\circ}$ 6, juin 1939, p. 56.

35. Arch. mun. Pantin, M21, Le point d'eau de Pantin, 1935.

\section{RÉSUMÉS}

En 1935, à l'occasion du projet de construction d'une usine élévatoire et de traitement des eaux par la C.G.E, la mairie de Pantin décide d'édifier à proximité une piscine municipale. Elle serait alimentée par les eaux chaudes extraites d'un des puits avoisinants. Le maire de l'époque, Charles Auray, passe commande à son fils, jeune architecte de vingt-quatre ans et lui adjoint un ingénieur plus expérimenté, Jean Molinié. Les deux bâtiments possèdent une parenté architecturale certaine, paradoxalement accentuée par les dernières modifications effectuées sur les façades de l'usine. Tout en récapitulant les traits propres aux piscines de l'époque, la piscine de Pantin emprunte sans complexe aux réalisations d'une des personnalités les plus marquantes du mouvement moderne néerlandais, l'architecte Willem-Marinus Dudok

In 1935, while the construction of a sewage pumping and treatment plant was planned by the administration of the French water utility, the town council of Pantin decided to have a municipal swimming pool built nearby. It would be supplied with warm waters drawn from one of the neighbouring wells. The mayor of the time, Charles Auray, commissioned his son, as a twenty-four-year-old architect, and appointed Jean Molinié, as a more experienced engineer, to him. The two buildings were definitely architecturally related, which would be paradoxically emphasized by the eventual alterations of the factory façades. Combining all the features characteristic of the contemporary swimming pools, the swimming pool of Pantin freely borrows from the works of one of the most outstanding personalities of the Dutch Modern Movement, the architect Willem-Marinus Dudok.

Die Compagnie Générale des Eaux (Gesellschaft für Wasserversorgung) plant 1935 die Errichtung einer Pumpen- und Abwasserreinigungsanlage in Pantin. Sogleich entschließt sich die Stadtverwaltung in deren Nähe ein städtisches Schwimmbad zu bauen, welches mit Warmwasser durch eine nahe artesische Quelle versorgt werden soll. Der damalige Bürgermeister Charles Auray beauftragt damit seinen Sohn, einen 24 jährigen jungen Architekten, und den erfahrenen Bauingenieur Jean Molinié als Mitarbeiter. Die Kläranlage und das Schwimmbad führen eine gewisse architektonische Verwandschaft vor Augen, die besonders deutlich wird nach den Veränderungen an den Fassaden der Abwasserreinigungsanlage. An dem Schwimmbad von 
Pantin lassen sich zwar alle Charakterzüge der zeitgenössischen Schwimmanstalten erkennen, aber auch der Einfluss der Werke des Architekten Willem-Marinus Dudok, einer bedeutenden Figur der niederländischen modernen Architektur

INDEX

Index chronologique : XXe siècle

Mots-clés : piscine

Keywords : swimming pool

Schlüsselwörter : Schwimmbad

\section{AUTEUR}

\section{CHRISTELLE INIZAN}

Christelle Inizan, née en 1964 à Paris, est titulaire d'une maîtrise d'histoire de l'art sur Dubuffet et son rapport à la matière, université de Paris I. Elle a poursuivi un cursus en art contemporain à Paris IV. Après avoir été journaliste en presse jeune, enseignante en histoire de l'art en lycée, elle entre en 1991 au ministère de la culture à la conservation régionale des monuments historiques d'île-de-France. Chargée d'études documentaires, elle a instruit plus de 80 protections au titre des monuments historiques, a donné plusieurs conférences notamment à l'École d'architecture de Versailles et à l'Institut national du patrimoine. Elle a participé à de nombreuses publications consacrées à l'architecture en Île-de-France. Adresse électronique:

christelle.inizan@culture.gouv.fr 